\title{
Buenas prácticas aplicadas a la adquisición de competencias diagnósticas clínicas en el ámbito del lenguaje
}

\author{
Best practices applied to the acquisition of clinical diagnostic skills in the \\ field of language
}

\author{
Calleja-Reina, M. ${ }^{1}$, Luque Liñán, M. L. ${ }^{1}$, Rodríguez Santos, J. M. ${ }^{1}$, Ferrer Urbano, J. ${ }^{2}$ y León Carrión, J. ${ }^{3}$ \\ emarinac@uma.es,mlluque@uma.es,jmiguel.rodriguez@uma.es,ferrer@lcc.uma.es. y leoncarrion@us.es \\ ${ }^{1}$ Departamento Psicología Básica \\ Universidad de Málaga \\ Málaga, España \\ ${ }^{3}$ Departamento de Psicología Básica. \\ Universidad de Sevilla \\ Sevilla, España
}

\begin{abstract}
Resumen En el presente trabajo se presenta una herramienta informática para el aprendizaje virtual simulado de competencias diagnósticas clínicas en el ámbito de las buenas prácticas en Ciencias de la Salud. La herramienta denominada LATS (Language Assessment Training System) pretende capacitar a los discentes del Grado en Logopedia para el diagnóstico clínico en el área del lenguaje y de la comunicación a partir de casos clínicos virtuales. Los casos clínicos virtuales cuentan con una serie de ventajas: se pueden realizar sin limitación de horarios, se pueden solicitar información de cada una de las fases del proceso diagnóstico tantas veces como consideremos oportuno, los datos de los tests estandarizados se encuentran a disposición del usuario siempre que los necesite. La herramienta dispone de 5 fases que son: motivo de consulta, anamnesis, hipótesis de partida, pruebas diagnósticas, diagnóstico final. La herramienta LATS propicia las buenas prácticas, ya que mejora el rendimiento, está fundamentada en una sólida experiencia previa y se plantea desde un enfoque innovador.
\end{abstract}

Palabras clave: Buenas prácticas, competencias diagnósticas, casos clínicos virtuales, enseñanza simulada, Ciencias de la Salud.

\begin{abstract}
This paper shows a computer tool for simulated virtual learning of clinical diagnostic competences in the field of good practices in Health Sciences. The tool called LATS (Language Assessment Training System) aims to train the students of the degree of Speech and Language Pathology for the clinical diagnosis in language and communication areas. The tool has 5 phases that are: reason for consultation, anamnesis, starting hypothesis, diagnostic tests, final diagnosis. Virtual clinical cases have a number of advantages: they can be performed without limitation of schedules, information can be requested from each of the phases of the diagnostic process as many times as we consider appropriate, the data of the standardized tests are available to the user whenever he need them. This tool promotes best practices, i.e. it improves performance, it is based on solid previous experience and it is considered from an innovative approach.
\end{abstract}

Keywords: best practices, diagnostic competencies, virtual clinical cases, simulated learning, Health Sciences

\section{INTRODUCCIÓN}

La adquisición de competencias clínicas para el diagnóstico es un proceso complejo que aborda la educación superior en el campo de las Ciencias de la Salud y en particular, en el ámbito de la Logopedia. El currículum universitario proporciona conocimientos y procedimientos a partir de los cuales los discentes demuestran a los profesores cómo resolverían problemas clínicos y posteriormente ponen en práctica este saber adquirido en situaciones reales en los Prácticum y en los inicios de su actuación profesional. Estas demostraciones de competencia para resolver los diagnósticos clínicos en Logopedia, con frecuencia se realizan mediante casos simulados que responden a los contenidos de cada asignatura individualmente $\mathrm{y}$ en escasas ocasiones los discentes se enfrentan a la toma de decisiones en cada una de las fases del proceso diagnóstico tal y como se produce en contexto clínico, integrando los conocimientos adquiridos parcialmente en cada asignatura del currículum. Para contribuir a que los discentes mejoren y demuestren su competencia para resolver diagnósticos clínicos en el ámbito de la Logopedia se ha construido una herramienta denominada LATS (Language Assessment Training System).

Aquellas prácticas educativas que permiten que el discente alcance contenidos, objetivos y competencias previstas se denominan buenas prácticas docentes (en adelante BPD) (Cabero y Romero (2010). Las BPD mejoran las prácticas actuales, desarrollan enfoques nuevos y variados además de posibilitar el aprendizaje activo de los discentes (Huber, 2008). De Pablos y Jiménez (2007) definen estas prácticas como actividades que proporcionan un cambio en la construcción de los conocimientos, en la configuración de nuevos entornos de enseñanza y aprendizaje y en la 
transformación de la cultura de la docencia (Durán Rodríguez y Estay-Niculcar, 2016). Epper \& Bates (2004) indican que las BPD han de estar fundamentadas en una experiencia sistematizada, documentada y experimentada, han de partir de un enfoque innovador y deben promover la aplicación de métodos de excelencia basados en la innovación y deben ser valoradas por los implicados y transferibles a otros contextos (Zabalza Beraza,2012).

El LATS es una herramienta que pretende facilitar la adquisición e incrementar las competencias para el diagnóstico en estudiantes universitarios de Logopedia siguiendo el modelo de BPD. Concretamente esta herramienta cuenta con un conjunto de casos clínicos virtuales que simulan la realidad que puede aparecer en la práctica clínica de los profesionales sanitarios. La metodología utilizada para dicho entrenamiento virtual-simulado consiste en el afrontamiento y la resolución de casos clínicos muy parecidos a los que se pueden encontrar en su desempeño profesional. La ventaja de emplear casos clínicos virtuales es la disponibilidad de los mismos en cualquier momento y tantas veces como considere el estudiante necesario. Cuando el discente emplea la herramienta LATS, su rol se modifica, y adquiere un papel de agente activo en la resolución de la situación clínica a la que tiene que dar respuesta. Además, la herramienta dispone de un proceso de retroalimentación, gracias a la cual, el discente va adquiriendo competencias para hacer frente a los retos profesionales de forma autónoma y activa. Además, se encuentra alojada en una máquina de la Universidad de Málaga (https://www.lats.uma.es/), lo que permite que los discentes pueden utilizarla desde las instalaciones de la UMA cuando lo consideren conveniente, sin el perjuicio de las limitaciones espacio-temporales de los casos clínicos con personas reales.

En resumen, el LATS es una herramienta diseñada para transformar la forma de enseñar de los docentes. Se ha utilizado en experiencias sistemáticas y documentadas (Calleja, Luque y Rodríguez, 2019) y ha sido valorada positivamente por los participantes en la experiencia. Por todo ello, el LATS constituye una aportación a las BPD en Educación Superior

\section{CONTEXTO}

La herramienta LATS, como se ha comentado, proporciona una oportunidad práctica para entrenar la competencia en diagnóstico clínico para los futuros profesionales del ámbito del lenguaje (Calleja, Luque y Rodríguez, 2018).

En otras disciplinas de Ciencias de la Salud existen programas detallados de entrenamiento en competencias diagnósticas clínicas (por ejemplo, los programas en Medicina como el Mini-Clinical Evaluation Exercise o mini-CEX) (para una revisión ver Pelgrim, Kramer, Mokkink, Van den Elsen, Grol y Van der Vleuten, 2011), mientras que para estudiantes de Logopedia no existe este tipo de herramientas para entrenar competencias diagnósticas.

La herramienta LATS se ha empleado con estudiantes de $3^{\circ}$ y $4^{\circ}$ curso del grado de Logopedia. Se ha desarrollado al amparo de sucesivas Convocatorias Competitivas de Proyectos de Innovación Educativa del Servicio de Formación e Innovación, dependiente del Vicerrectorado de Personal Docente e Investigador de la Universidad de Málaga ${ }^{1}$.

El objetivo del presente trabajo es mostrar la utilidad de la herramienta LATS para el entrenamiento en destrezas diagnósticas en el ámbito de la Logopedia como una BPD.

\section{DESCRIPCIÓN}

El LATS es una herramienta diseñada por los tres primeros autores de este trabajo, que pretende guiar al discente en el proceso de toma de decisiones, simulando las fases que utiliza un experto clínico en su funcionamiento cotidiano:

$1^{\circ}$. Seleccionar la información necesaria y relevante que necesita de la anamnesis, o entrevista inicial, descartando la irrelevante.

$2^{\circ}$. Formular las hipótesis clínicas apropiadas que guíen la evaluación para identificar la etiqueta diagnóstica

$3^{\circ}$. Seleccionar las herramientas diagnósticas adecuadas para la hipótesis clínica prevista y la obtención del perfil lingüístico.

$4^{\text {o }}$. Realizar un diagnóstico que abarque todas las áreas deficitarias del paciente, identificando la etiqueta diagnóstica con las características correspondientes a cada caso.

$5^{\circ}$. Justificar el diagnóstico aportando una reflexión razonada sobre los resultados obtenidos en el proceso diagnóstico y la importancia de cada resultado en el proceso de diagnóstico, es decir, de asignación de la etiqueta.

El estudiante puede acceder a la aplicación tantas veces como considere oportuno, modificando hipótesis, pruebas y diagnóstico final $y$ el programa le proporciona una retroalimentación de la idoneidad del proceso. El proceso se describe a continuación:

$1^{\circ}$. Se accede a la ubicación de la herramienta en el portal virtual de la Universidad de Málaga introduciendo el usuario y la clave de acceso proporcionada. Se accede al menú principal de la aplicación y se selecciona "Realizar un diagnóstico" y aparece un listado con una serie de motivos de consulta disponibles y se elige uno de ellos. A continuación, se seleccionar la información necesaria y relevante que necesita de la anamnesis, o entrevista inicial, descartando la irrelevante. Al seleccionar el motivo de consulta se inicia la $1^{\text {a }}$ fase del proceso. La herramienta permite valorar la relevancia de las elecciones del estudiante.

$2^{\circ}$. En la segunda fase el alumno debe seleccionar la hipótesis de partida de su evaluación. El docente en esta fase obtiene información sobre la capacidad de ajuste que tiene el estudiante entre la información obtenida en la anamnesis y el planteamiento de la primera Hipótesis diagnóstica.

$3^{\circ}$. En la fase $3^{\mathrm{a}}$ se presenta un listado de pruebas entre las que el estudiante debe elegir las apropiadas a la hipótesis diagnóstica inicial y las características del caso.

$4^{\circ}$. En la $4^{\text {a }}$ fase el alumno analiza los resultados de las diferentes pruebas seleccionadas cuyos resultados se han acumulado en un documento en formato pdf, a la vez que en la zona de toma de decisiones se le pide que seleccione el

\footnotetext{
${ }^{1}$ La dotación económica para llevar a cabo esta herramienta
} ha sido de 2.080 euros (PIE 013-017 y PIE 017-138). 
diagnóstico apropiado para el caso y su confirmación para pasar a la siguiente fase. El docente en esta fase valora la toma de decisiones realizada.

$5^{\circ}$. En la quinta fase el estudiante debe valorar la importancia de cada dato en la selección del diagnóstico realizada. El docente valora la capacidad de razonamiento clínico en la elección del diagnóstico y su justificación con los resultados obtenidos, diferenciando niveles en el razonamiento realizado por los estudiantes.

Por último, se proporciona al estudiante una valoración numérica y cualitativa (muy bajo, bajo, medio, alto y muy alto) de cada experiencia realizada con el LATS. Además, se ofrece retroalimentación acerca de las pruebas seleccionadas para justificar las puntuaciones que ha alcanzado, así como para mejorar sus conocimientos clínicos. El estudiante puede descargar el documento en formato pdf con la información acumulada a modo de informe de resultados para continuar su aprendizaje. Por último, se realiza un cuestionario de satisfacción de la experiencia.

\section{Resultados}

La herramienta ha sido probada con dos grupos de estudiantes de tercer $(\mathrm{n}=18)$ y cuarto curso de logopedia $(\mathrm{n}=10)$. Tras la utilización del LATS durante tres ensayos, los alumnos de $3^{\circ}$ incrementan sus puntuaciones (ver Ilustración 1). En el primer ensayo alcanzaron una puntuación media de 22,80 , en el segundo ensayo registramos un ascenso de las puntuaciones medias hasta 32,32 , y, en el tercer ensayo la puntuación media se incrementa hasta los 42,46 puntos.

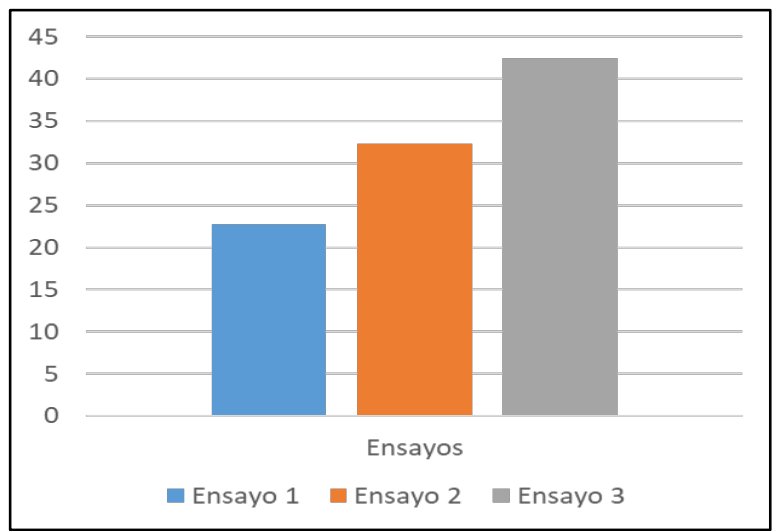

Ilustración 1. Evolución de las puntuaciones en $3^{\circ}$ curso en los tres momentos de ensayo

La comparación de las puntaciones finales alcanzadas por los alumnos de $3^{\circ}$ y $4^{\mathrm{a}}$ indican un incremento a favor de los alumnos que están finalizando los estudios de grado (ver Ilustración 2).

A la vista de las puntuaciones finales alcanzadas por los discentes en esta experiencia, observamos una mejora en la toma de decisiones clínicas con el entrenamiento sucesivo con el LATS.

En relación a la encuesta de opinión, los estudiantes consideran que la herramienta es útil y accesible en el $80 \%$ de los casos y está bien estructurada y es adecuada para el aprendizaje del proceso de toma de decisiones para el diagnóstico a partir de la identificación de perfiles comunicativos y lingüísticos de los pacientes.

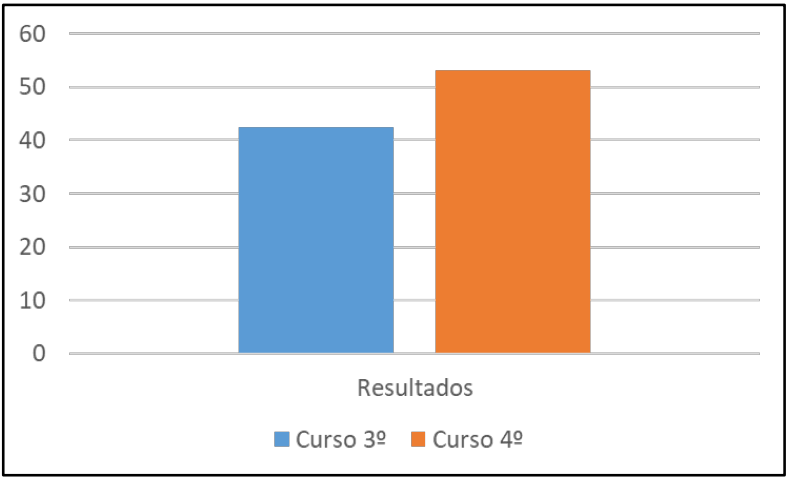

Ilustración 2. Comparación de las puntuaciones finales de alumnos de $3^{\circ}$ y $4^{\circ}$ curso del Grado en Logopedia.

\section{CONCLUSIONES}

Esta herramienta ha sido diseñada para entrenar y evaluar las competencias clínicas diagnósticas en el ámbito de la Logopedia y de la Psicología, como una aplicación que facilite y mejore las buenas prácticas en el ámbito del diagnóstico de los problemas del Lenguaje y de la Comunicación. La experiencia con los alumnos de $3^{\circ}$ y $4^{\circ}$ del Grado de Logopedia ha confirmado que mejoran sus destrezas en la resolución del caso propuesto con la práctica y valoran positivamente la experiencia. Por lo tanto, el LATS es una herramienta válida para el entrenamiento en competencias y cumple diferentes principios de las buenas prácticas en la docencia universitaria:

a) Los alumnos mejoran su competencia clínica en diagnóstico.

b) Se realiza en un entorno virtual. El LATS está colgado en la plataforma virtual de la Universidad de Málaga. El acceso a los casos se realiza mediante un ordenador.

c) Se ha utilizado en docencia para asignaturas de diferentes cursos del currículum del Plan de estudios de Grado en Logopedia.

d) Se han realizado diferentes experiencias sistemáticas y documentadas que pueden ser replicadas con alumnos de diferentes cursos del Grado de Logopedia (Calleja, Luque y Rodríguez, 2018) además de la experiencia expuesta.

e) Propone una experiencia docente innovadora aplicada a la práctica clínica diagnóstica en el ámbito del Lenguaje y la Comunicación dada la ausencia de herramientas de esta naturaleza aplicada a la adquisición de competencias en diagnóstico clínico en Logopedia que, además, es bien valorada por los participantes.

\section{AgradeCIMIENTOS}

Este trabajo ha sido financiado por el Vicerrectorado PDI, Servicio de Formación de la Universidad de Málaga en la Convocatoria de Proyectos de Innovación Educativa (PIE 2017/138) de la Universidad de Málaga. Campus de Excelencia Andalucía Tech. 


\section{REFERENCIAS}

Calleja Reina, M.; Rodríguez Santos, J. M. \& Luque Liñán, M.L. (2018). Utilidad de una herramienta informática para la adquisición de competencia en razonamiento clínico en logopedia. Educación Médica, 19(3),162-165.

Calleja Reina, M.; Rodríguez Santos, J. M. \& Luque Liñán, M.L. (2019). LATS, una herramienta TIC para la enseñanza proactiva de competencias en diagnóstico clínico en el ámbito de los problemas del lenguaje. En E. Postigo-Pinazo (Coord). Nuevas tecnologías, procesos cognitivos y estrategias para la optimización de las competencias del traductor e intérprete. Frank \& Timme: Berlín.

Cabero, J. y Romero R. (2010). Análisis de buenas prácticas del e-learning en las universidades andaluzas. Revista Electrónica Teoría de la Educación: Educación y Cultura en la Sociedad de la Información, 11(1), 283309.

De Pablos Pons, J. y Jiménez Cortés, R. (2007). Buenas prácticas con TIC apoyadas en las Políticas Educativas: claves conceptuales y derivaciones para la formación en competencias ECTS. Revista Latinoamericana de Tecnología Educativa, 6 (2), 15-28
Durán Rodríguez, R. y Estay-Niculcar, C.A. (2016). Las buenas prácticas docentes en la educación virtual universitaria. Revista de Docencia Universitaria, 14 (2), $159-186$

Epper, R. M. \& Bates, A. W. (2004). Enseñar al profesorado cómo utilizar la tecnología. Buenas prácticas de instituciones líderes. Barcelona: Editorial UOC

Huber, G. L. (2008). Aprendizaje activo y metodologías educativas. Revista de Educación, $N^{o}$ extraordinario 1, 59-84.

Keengwe, J. \& Kidd, T.T. (2010).Towards Best Practices in Online Learning and Teaching in Higher Education. MERLO. Journal of Online Learning and Teaching, Vol. 6 (2), pp.533-541

Pelgrim, E. A., Kramer, A. W. M., Mokkink, H. G., Van den Elsen, L., Grol, R. P. T. M., \& Van der Vleuten, C. P. M. (2011). In-training assessment using direct observation of single-patient encounters: a literature review. Advances in health sciences education, 16(1), 131-142.

Zabalza Beraza, M.A. (2012). El estudio de las "buenas prácticas" docentes en la enseñanza universitaria. Revista de Docencia Universitaria, 10 (1), 17-42. 